\title{
Adaptive Modeling of Complex Packaging Geometries Using Haar-based MRTD Algorithms
}

\author{
B.McGarvey, D.Staiculescu, M.Tentzeris and J.Laskar \\ daniela@ece.gatech.edu, etentze@ece.gatech.edu,joy.laskar@ece.gatech.edu \\ Packaging Research Center \\ School of Electrical and Computer Engineering \\ Georgia Institute of Technology \\ Atlanta, GA 30332-0250
}

\begin{abstract}
The Haar-based MRTD algorithm is applied to the design and optimization of complex 3D RF Packaging geometries. Significant numerical aspects concerning the implementation of Boundary Conditions and the thresholding of the wavelet coefficients are discussed in detail and guidelines for the efficient modeling of a practical Flip-Chip package are derived.
\end{abstract}

\section{Multiresolution Time-Domain Techniques - Expansion Basis}

The MultiResolution Time-Domain (MRTD) algorithms have demonstrated unparallel properties when applied to the analysis of structures with medium or large computational domains [1-6]. Through a two-fold expansion of the fields in scaling and wavelet functions with respect to time/space, memory and execution time requirements are minimized while a high resolution in areas of strong field variations or field singularities is achieved through the use of sufficiently large number of wavelet resolutions. The major advantage of the MRTD algorithms is their capability to develop real-time time and space adaptive grids through the efficient thresholding of the wavelet coefficients.

Various expansion basis have been utilized for the implementation of the MRTD algorithms. The Battle-Lemarie basis offers a reduction in memory by 2-3 orders of magnitude for 3D structures. Nevertheless, the entire-domain character of these functions adds a significant computational overhead in the approximation of the field derivatives in Curl Maxwell equations. In addition, Hard Boundaries (e.g. PEC's) cannot be applied directly by zeroing out the appropriate field components; image theory has to be implemented to account for the neighboring cells' contribution. Due to their compact support, Haar expansion basis functions (Fig.1) provide schemes that are similar to the FDTD algorithm that can be derived using pulse basis. They do not provide the drastical economies of the entire-basis schemes, but can be implemented in a much simpler way and maintain the adaptive feature.

\section{MRTD Scheme with Arbitrary Wavelet Resolutions}

For simplicity, the 1D MRTD scheme for TEM propagation will be presented. It can be extended to 2D and 3D in a straightforward way. The Electric $\left(\mathrm{E}_{\mathrm{x}}\right)$ and the Magnetic $\left(\mathrm{H}_{\mathrm{y}}\right)$ fields are displaced by half step in both time- and space-domains (Yee cell formulation) and are expanded in a summation of scaling $(\phi)$ and wavelet $(\psi)$ functions in space and scaling components in time. For example, $\mathrm{E}_{\mathrm{x}}$ is given by $\mathrm{E}_{\mathrm{x}}(\mathrm{z}, \mathrm{t})=\Sigma_{\mathrm{m}, \mathrm{i}}\left\{{ }_{\mathrm{m}} \mathrm{E}_{\mathrm{x}, \mathrm{i}}{ }^{\phi} \quad \phi_{\mathrm{i}}(\mathrm{z})+\sum_{\mathrm{r}=0->\mathrm{r} \max } \sum_{\mathrm{ir}=1->2}{ }^{\mathrm{r}}\right.$ ${ }_{\mathrm{m}} \mathrm{E}_{\mathrm{x}, \mathrm{i}}{ }^{\psi}$ r,ir $\left.\quad \psi_{\mathrm{i}}^{\mathrm{r}, \mathrm{ir}}(\mathrm{z})\right\} \quad \phi_{\mathrm{m}}(\mathrm{t})$, where $\phi_{\mathrm{i}}(\mathrm{z})=\phi(\mathrm{z} \Delta \mathrm{z}-\mathrm{i})$ and $\psi_{\mathrm{i}}^{\mathrm{r}, \mathrm{ir}}(\mathrm{z})=2^{\mathrm{r} / 2} \psi_{0}\left(2^{\mathrm{r}}(\mathrm{z} \Delta \mathrm{z}-\mathrm{ir})-\mathrm{i}\right)$ represent the Haar scaling and r-resolution wavelet functions located inside the $i$-cell. The conventional notation ${ }_{m} E_{x, i}$ is used for the Electric field component at time $\mathrm{t}=\mathrm{m} \Delta \mathrm{t}$ and $\mathrm{z}=\mathrm{i} \Delta \mathrm{z}$, where $\Delta \mathrm{t}$ and $\Delta \mathrm{z}$ are the time-step and the spatial cell size respectively. The notation for $\mathrm{H}_{\mathrm{y}}$ is similar. 
Substituting $\mathrm{E}_{\mathrm{x}}, \mathrm{H}_{\mathrm{y}}$ in the TEM equations and applying Galerkin technique derives MRTD equations. Dielectric Interfaces are modeled through the discretization of the constitutive relationship $\mathrm{D}=\varepsilon \mathrm{E}$ and the solution of a matrix equation involving all scaling and wavelet components with domains containing the interface.

Due to the finite-domain nature of the expansion basis, the Hard Boundary conditions (Perfect Electric/Magnetic Conductor) can be easily modeled. For example, if a P.E.C. exists at the $\mathrm{z}=i \Delta \mathrm{z}$, then the scaling $\mathrm{E}_{\mathrm{x}}$ coefficient for the $i$ cell has to be set to zero for each time-step $m$ since the position of the conductor coincides with the midpoint of the domain of the scaling function. Nevertheless, the 0-resolution wavelet for the same cell has the value of zero at its midpoint; thus its amplitude does not have to be set to zero. To enforce the physical condition that the electric field values on either side of the conductor are indpendent from the files on the other side, TWO 0-resolution wavelet $\mathrm{E}_{\mathrm{x}}$ coefficients have to be defined. The one (on the one side of P.E.C.) will depend on $\mathrm{H}_{\mathrm{y}}$ values on this side only and the other (on the other side of P.E.C.) will depend on $\mathrm{H}_{\mathrm{y}}$ values on that side only. Wavelet coefficients of higher-resolution with domains tangential to the position of P.E.C. have to be zeroed out as well.It can be easily observed that for Wavelet Resolutions up to $r_{\max }$, $2^{\text {rmax+1 }}$ coefficients have to be calculated per cell per field component instead of one component in the conventional F.D.T.D.

The fact that the wavelet coefficients take significant values only for a small number of cells that are close to abrupt discontinuities or contain fast field variations allows for the development of a dynamically adaptive gridding algorithm. One thresholding technique based on absolute and relative thresholds offers very significant economy in memory while maintaining the increased resolution in space where needed. For each time-step, the values of the scaling coefficients are first calculated for the whole grid. Then, wavelet coefficients with resolutions of increasing order are updated. As soon as all wavelet components of a specific resolution of a cell have values below the
Absolute Threshold (that has to do with the numerical accuracy of the algorithm) or below a specific fraction (Relative Threshold) of the respective scaling coefficient, no higher wavelet resolutions are updated and the simulation moves to the update of the wavelet coefficients of the next cell. In this way, the execution time requirements are optimized, since for areas away from the excitation or discontinuities, only the scaling coefficients need to be updated. This is a fundamental difference with the conventional F.D.T.D. algorithms that cannot provide a dynamical time- and space- adaptivity even with grids of variable cell sizes (static adaptivity).

\section{Flip-Chip Geometry - Validation}

The computational advantages offered by MRTD allow for the accurate modeling of practical complex Packaging structures with fine dielectric and conductor details, such as the FlipChip configuration [7], that would require an unrealistic execution time using conventional (FDTD) techniques. Figures 2a, $2 \mathrm{~b}$ show the Flip-Chip prototype that has been fabricated in Georgia Tech Packaging Research Center. Flip chip test structures have been fabricated on 254 $\mu \mathrm{m}$ thick alumina. The gold thermocompressed bumps are $25 \mu \mathrm{m}$ in height and $70 \mu \mathrm{m}$ in diameter. On-wafer S-parameter measurements have been performed on a HP $85109 \mathrm{C}$ network analyzer using $150 \mu \mathrm{m}$ pitch coplanar waveguide probes. Comparison between MRTD, FEM and measurements of the test structure are presented in Figure 3.

\section{Conclusion}

Numerical aspects concerning the application of the Haar-based MRTD to the design and optimization of complex RF Packaging geometries have been discussed. MRTD has the potential to be a very efficient design tool for the optimization of state-of-the-art wireless systems components, since it demonstrates significant economy in computational resources and adaptive resolution. A conventional Flip-chip geometry is used as the test vehicle for the validation of this algorithm. 


\section{Acknowledgement}

The authors would like to acknowledge the support of NSF Packaging Research Center .

\section{References}

[1] E.Tentzeris, R.Robertson, A.Cangellaris and L.P.B. Katehi, "Space- and TimeAdaptive Gridding Using MRTD”, Proc. MTT-S 1997, pp.337-340.

[2] M.Krumpholz, L.P.B.Katehi, "MRTD: New Time Domain Schemes Based on Multiresolution Analysis", IEEE Trans. Microwave Theory and Techniques, vol.44, no.4, pp.555-561, April 1996.

[3] E.Tentzeris, J.Harvey and L.P.B.Katehi, "Time Adaptive Time-Domain Techniques for the Design of Microwave Circuits",
IEEE Microwave and Guided Wave Letters, Vol.9, No.3, pp.96-98, 1999.

[4] K.Goverdhanam, E.Tentzeris, M.Krumpholz and L.P.B. Katehi, "An FDTD Multigrid based on Multiresolution Analysis", Proc. AP-S 1996, pp.352-355.

[5] M.Fujii, W.J.R.Hoefer, "Formulation of a Haar-wavelet based Multiresolution Analysis similar to the 3-D FDTD Method", Proc. MTT-S 1998, pp.1393-1396.

[6] C.Sarris and L.P.B.Katehi, "Multiresolution Time Domain (MRTD) Schemes with Space-Time Haar Wavelets", Proc. MTT-S 1999, pp.1459-1462

[7] K. Boustedt, "GHz flip chip - an overview", 1998 Electronic Components and Technology Conference", Seattle, WA, pp. 1280-1285.

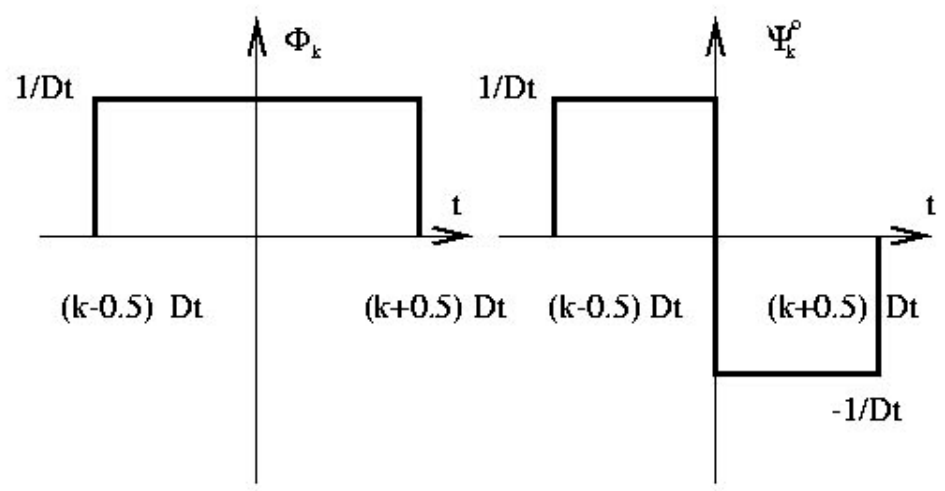

Fig.1 Haar Expansion Basis.

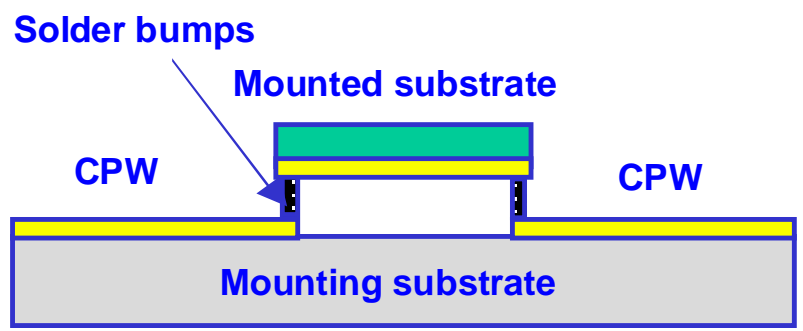

PW - CPW transition

Fig.2a Side View of Flip-Chip Geometry. 


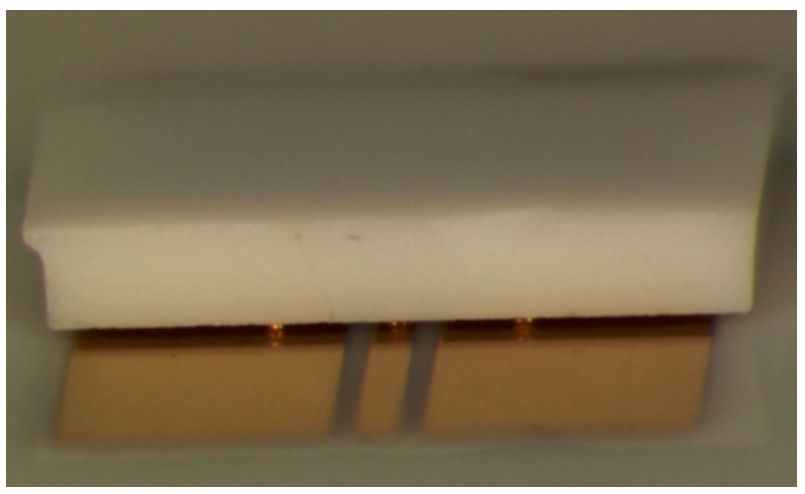

Fig.2b Picture of Fabricated Test Structure

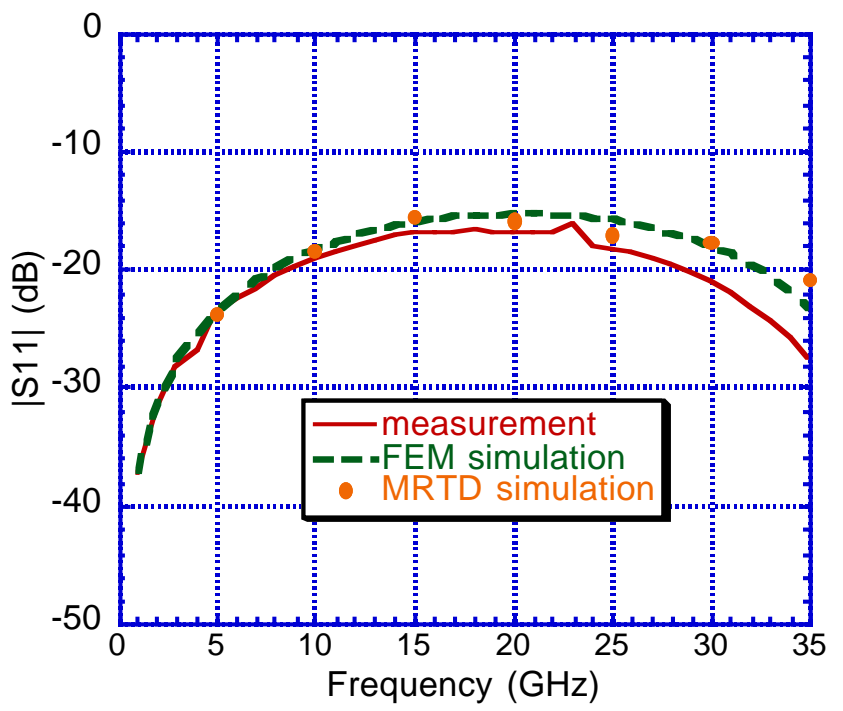

Figure 3. Verification with FEM and Test Structure Measurements 